then every 3 months after RFA to assess for the development of treatment-related complications.

Results A total 115 procedures were performed successfully. No death related to the technique. There were 3 cases with early complication (1 diaphragm perforation, 1 lesion abcess, 1 pleural effusion,) and tumour seeding developed in 2 patients. The rate of Post RFA syndrome was $67 \%$, but most of the symptoms were transient and self-limited.

Conclusions RFA using Cool-tip is a safe treatment for patients HCC.

\section{IDDF2018-ABS-0080 RISK FACTORS FOR LOCAL RECURRENCE IN THE TREATMENT OF RADIOFREQUENCY ABLATION WITH COOL-TIP ELECTRODE FOR HCC PATIENTS}

${ }^{1}$ Nguyen Thi Thu Huyen*, ${ }^{2}$ Mai Hong Bang, ${ }^{2}$ Tran Van Riep, ${ }^{2}$ Nguyen Tien Thinh. ${ }^{1}$ Thai Nguyen University of Medicine and Pharmacy, Vietnam; ${ }^{2} 108$ Central Hospital, Vietnam

\subsection{6/gutjnl-2018-IDDFabstracts.203}

Background Radiofrequency ablation (RFA) is one of the curative therapies for HCC patients. However, post-RFA local recurrence is a major factor limiting the outcome. The aim of this study was to evaluate the recurrent rate and analyse the risk factors for local recurrence of percutaneous Radiofrequency Ablation using Cool-tip electrode for the treatment of HCC.

Methods A prospective study involved 82 cirrhotic HCC patients (mean tumour size: 3,2 $\pm 1,1 \mathrm{~mm}$ ) underwent percutaneous RFA using Cool-tip RF electrode (COOL-TIP E SERRIES, COVIDIEN) at the 108 Hospital, from September 2012 to November 2017. We use single gauge, cluster, or multiple electrodes with an exposed needle tip of variable length $(2,3$ or $4 \mathrm{~cm}$ ). The rate of recurrence was recorded, and the prognostic factors for the tumour local recurrence were determined.

Results There were $37 / 75$ of patients presented recurrence after achieved complete response, including local recurrence in $11 / 75(14.7 \%)$ new nodule recurrence in 16/75 (21.3\%) and both local and new nodule recurrence in $7 / 75$ (9.3\%). The mean time of recurrence was 23 months (12-45 months). Tumour size $(3 \mathrm{~cm}-5 \mathrm{~cm})$, tumour location (close to vascular), size of ablative margin $(<0.5 \mathrm{~cm})$, high serum AFP level had a significant adverse prognostic factor for local tumour recurrence.

Conclusions Although RFA using Cool-tip is an effective treatment for local tumour control in HCC patients, the long term result depends on some prognostic factors before treatment.

\section{IDDF2018-ABS-0081 GRADUALLY DECREASING OF SERUM HBCRAG IN REAL-WORLD CHRONIC HEPATITIS B PATIENTS RECEIVING LONG- TERM NUCLEOT(S)IDE ANALOGUES-BASED THERAPY}

Menglan Wang*, Enqiang Chen, Chuanming Tao, Juan Liao, Taoyou Zhou, Juan Wang, Libo Yan, Dongbo Wu, Lingyao Du, Hong Tang. West China Hospital of Sichuan University, China

10.1136/gutjnl-2018-IDDFabstracts.204
Background This study aimed to investigate the detailed dynamics of $\mathrm{qHBcrAg}$ and influence factors of long-term qHBcrAg in a cohort of patients who had received over 8 years of continuous NAs therapy.

Methods This was a retrospective study. All patients were recruited from our previous published study, who started therapy with NAs between 2007 and 2008. Serum HBsAg and HBcrAg levels were quantitatively measured at baseline, the sixth month and each year of follow-up, using the stored serum samples.

Results Among the 94 patients, serum qHBcrAg presented a gradually decreasing trend from baseline to year 8 , either in $\mathrm{HBeAg}$-negative or $\mathrm{HBeAg}$-positive patients. Good correlation of $\mathrm{qHBcrAg}$ and $\mathrm{qHBsAg}$ was observed at baseline, but this correlation weakened remarkably during treatment. Serum qHBcrAg.

Conclusions Serum HBcrAg levels are varied during the course of antiviral therapy, and $\mathrm{qHBcrAg}$ may serve as a new important tool for the management of CHB.

\section{IDDF2018-ABS-0083 NONALCOHOLIC FATTY LIVER DISEASE IS ASSOCIATED WITH INCREASED ATRIAL FIBRILLATION RISK IN AN ELDERLY CHINESE POPULATION: A CROSS- SECTIONAL STUDY}

${ }^{1}$ Yu Zhang $*$, ${ }^{2}$ Peifei Li, ${ }^{2} Y_{\text {Li Liu, }}{ }^{2}$ Yue Pan, ${ }^{1}$ Lu Xu, ${ }^{3}$ Min Miao, ${ }^{4}$ Zhongwei Zhu, ${ }^{5} \mathrm{Chengfu} \mathrm{Xu}$ ${ }^{2}$ Lei Xu. ${ }^{1}$ Ningbo University, College of Medicine, Ningbo, China; ${ }^{2}$ Department of Gastroenterology, Ningbo First Hospital, Ningbo, China; ${ }^{3}$ Department of Gastroenterology, The Affiliated Hospital of Ningbo University, School of Medicine, Ningbo, China; ${ }^{4}$ Department of Gastroenterology, Zhenhai Lianhua Hospital, Ningbo, China; ${ }^{5}$ Department of Gastroenterology, The First Affiliated Hospital, College of Medicine, Zhejiang University, Hangzhou, China

\subsection{6/gutjnl-2018-IDDFabstracts.205}

Background Atrial fibrillation and nonalcoholic fatty liver disease are two pathological conditions that are highly prevalent worldwide and share multiple CVD risk factors. There is rare research performed among elderly adults.

Aims We conducted this cross-sectional analysis of elderly adults ( $\geq 65$ years) to investigate the association between atrial fibrillation and nonalcoholic fatty liver disease.

Subjects We conducted a cross-sectional study of the elderly adults ( $\geq 65$ years old) who had undergone an annual physical examination at Zhenhai Lianhua Hospital, Ningbo, China in 2014. 1688 participants (930 males and 758 females) with a median age of 72 (68-76) years were included in this analysis. This study excluded the following participants: (1) those with unknown alcohol intake or excessive alcohol intake; (2) those with unknown BMI or BMI2; (3) those with incomplete basic physical data; (4) those with missing liver ultrasonic diagnosis; (5) those with unknown causes of chronic liver disease. This study was approved by the Hospital Ethics Committee. All the participants were verbally informed and agreed to participate in the study. Written informed consent was not required for the observational nature of the study. Methods We analysed clinical data of the elderly adults $(\geq 65$ years) who took health examination in Zhenhai Lianhua hospital, Ningbo, China in 2014.

Results 522 of the 1688 participants were diagnosed with nonalcoholic fatty liver disease, and 39 participants were confirmed as having atrial fibrillation. Nonalcoholic fatty liver disease was associated with risk factors for AF in the elderly Chinese population (OR 1.95, 95\% CI 1.03-3.69). 
Adjustments for age, gender, systolic blood pressure, fasting plasma glucose, $\gamma$-glutamyl transpeptidase, high-density lipoprotein, triglycerides, total cholesterol and albumin, nonalcoholic fatty liver disease and prevalent atrial fibrillation remained statistically significant (OR 2.76, 95\% CI 1.32-5.77).

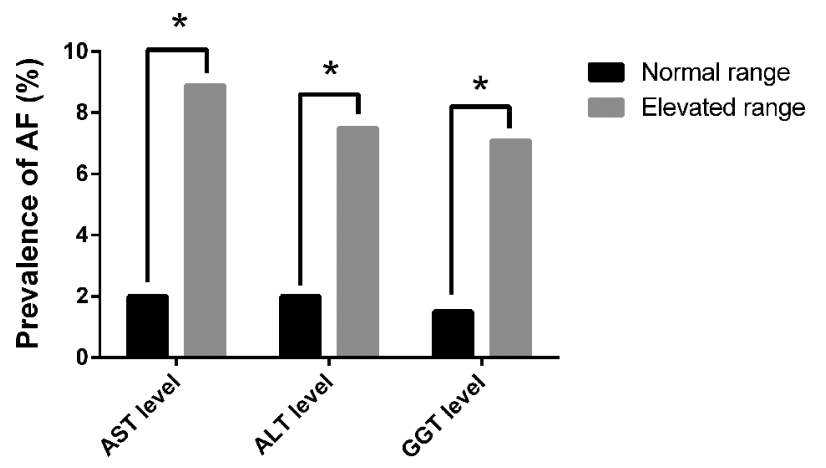

Abstract IDDF2018-ABS-0083 Figure 1

Conclusions Our results show that nonalcoholic fatty liver disease is associated with an increased risk of atrial fibrillation in an elderly Chinese population.

\section{IDDF2018-ABS-0098 PREOPERATIVE PREDICTION OF MICROVASCULAR INVASION IN HEPATOCELLULAR CANCER: A RADIOMICS MODEL USING GD-EOB-DTPA ENHANCED MRI}

${ }^{1}$ Mimi Tang*, ${ }^{2}$ Shi-Ting Feng, ${ }^{1}$ Sui Peng, ${ }^{3}$ Ming Kuang. 'Department of Gastroenterology, The First Affiliated Hospital of Sun Yat-sen University, Guangzhou, China; ${ }^{2}$ Department of Radiology, The First Affiliated Hospital of Sun Yat-sen University, China; ${ }^{3}$ Department of Liver Surgery, The First Affiliated Hospital of Sun Yat-sen University, China

\subsection{6/gutjnl-2018-IDDFabstracts.206}

Background Preoperative prediction of microvascular invasion (MVI) in patients with hepatocellular cancer (HCC) is important for surgery strategy making. We aimed to develop and validate a combined intratumoral, and peritumoral radiomics model based on Gadolinium-ethoxybenzyl-diethylenetriamine (Gd-EOB-DTPA) enhanced magnetic resonance imaging (MRI) for preoperative prediction of MVI in primary HCC patients. Methods This study included a training cohort of 110 HCC patients and a validating cohort of 50 HCC patients. All the patients underwent preoperative Gd-EOB-DTPA enhanced MRI examination and curative hepatectomy. The volumes of interest (VOIs) around the hepatic lesions including intratumoral and peritumoral regions were manually delineated in the hepatobiliary phase of MRI images, from which quantitative features were extracted and analysed. In the training cohort, the machine-learning method was applied to dimensionality reduction and selection of the extracted features.

Results The proportion of MVI-positive patients was 38.2\% and $40.0 \%$ in the training and validation cohort respectively. Supervised machine-learning selected ten features to establish a predictive model for MVI. The area under the receiver operating characteristic curve (AUC), sensitivity, specificity of the combined intratumoral and peritumoral radiomics model in the training and validation cohort were 0.85 (95\% confidence interval $[C I], 0.77-0.93), 88.2 \%, 76.2 \%$ and $0.83(95 \%$ CI, 0.71-0.95), 90.0\%, 75.0\%, respectively.

Conclusions We evaluate quantitative Gd-EOB-DTPA enhanced MRI image features of both intratumoral and peritumoral regions and provided an effective radiomics-based model for prediction of MVI in HCC patients, and may, therefore, assist clinicians to make precise treatment decision before the surgery.

\section{IDDF2018-ABS-0105 NO RESISTANCE TO TENOFOVIR ALAFENAMIDE DETECTED THROUGH 96 WEEKS OF TREATMENT IN PATIENTS WITH CHRONIC HEPATITIS B}

${ }^{1}$ Henry Lik-Yeun Chan*, ${ }^{2}$ Patrick Marcellin, ${ }^{3}$ Andrew L Cathcart, ${ }^{3}$ Neeru Bhardwaj, ${ }^{3}$ John F Flaherty, ${ }^{3}$ Anuj Gaggar, ${ }^{4}$ Namiki Izumi, ${ }^{5}$ Young-Suk Lim. ${ }^{1}$ Department of Medicine and Therapeutics, Institute of Digestive Disease and State Key Laboratory of Digestive Disease, The Chinese University of Hong Kong, Hong Kong; ${ }^{2}$ Service d'Hepatologie, Hopital Beaujon, Clichy, France; ${ }^{3}$ Gilead Sciences ,Foster City, CA, USA; ${ }^{4}$ Department of Gastroenterology and Hepatology, Musashino Red Cross Hospital, Tokyo, Japan; ${ }^{5}$ Department of Gastroenterology, Asan Medical Center, Seoul, Korea, South

\subsection{6/gutjnl-2018-IDDFabstracts.207}

Background Presented herein are the post Week 48 through Week 96 resistance analyses for 2 Phase 3 studies evaluating tenofovir alafenamide (TAF) versus tenofovir disoproxil fumarate(TDF) for the treatment of chronic hepatitis $\mathrm{B}(\mathrm{CHB})$ in HBeAg + and HBeAg- adults.

Methods HBV pol/RT population or deep sequencing was conducted for patients with $\geq 24$ weeks of treatment with viremia (HBV DNA $\geq 69 \mathrm{IU} / \mathrm{mL}$ ) at Week 96 or at early discontinuation post Week 48. Deep sequencing was conducted for patients with HBV DNA $>159 \mathrm{IU} / \mathrm{mL}$ and sequence changes at the consensus sequence level (15\%) are reported. Virologic breakthrough (VB) was defined as $\mathrm{HBV}$ DNA $\geq 69 \mathrm{IU} / \mathrm{mL}$ after achieving $<69 \mathrm{IU} / \mathrm{mL}$ or $\mathrm{a} \geq 1.0-\log _{10}$ increase from nadir. Phenotypic analysis using recombinant HBV in HepG2 cells was performed for VB patients who were adherent to study drug (plasma drug levels), patients with conserved site substitutions, or for polymorphic substitutions emergent in $>1$ patient.

Results 1298 patients were randomised (TAF: $n=866$; TDF: $\mathrm{n}=432$ ). A similar percentage of patients in the TAF or TDF arms qualified for sequence analysis post Week 48 through Week 96 of treatment (TAF: 10.5\%, TDF: 10.9\%). In the TAF arm, 87 patients qualified at Week 96: 32 had polymorphic site substitutions, and 9 had conserved site substitutions. In the TDF arm, 45 patients qualified at Week $9611 \mathrm{had}$ polymorphic site substitutions, and 2 had conserved site substitutions. At Week 96, a small percentage of patients experienced VB (TAF: 2.4\%, TDF: 3.0\%), and VB was often associated with documented study drug nonadherence (TAF: 22\%, TDF: 46\%). 27 patients qualified for phenotypic analysis post Week 48 through Week 96 (TAF: $n=19$, TDF: $n=8$ ) and no patient isolates tested showed a reduction in susceptibility to TAF or tenofovir, respectively. 